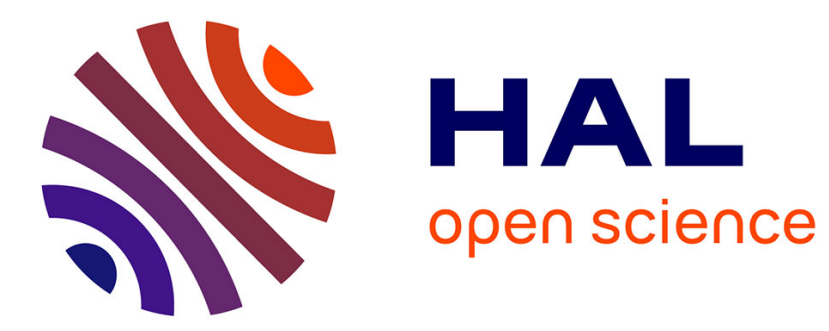

\title{
Tensile mechanics of polymeric foam ribbons
}

Aymeric Mouquet, Yacine Khidas, Xavier Chateau, Jean-Yvon Faou, Tamar

Saison, Olivier Pitois

\section{To cite this version:}

Aymeric Mouquet, Yacine Khidas, Xavier Chateau, Jean-Yvon Faou, Tamar Saison, et al.. Tensile mechanics of polymeric foam ribbons. Polymer Testing, 2020, 86, pp.106513. 10.1016/j.polymertesting.2020.106513 . hal-02883731

\section{HAL Id: hal-02883731 \\ https://hal-enpc.archives-ouvertes.fr/hal-02883731}

Submitted on 29 Jun 2020

HAL is a multi-disciplinary open access archive for the deposit and dissemination of scientific research documents, whether they are published or not. The documents may come from teaching and research institutions in France or abroad, or from public or private research centers.
L'archive ouverte pluridisciplinaire HAL, est destinée au dépôt et à la diffusion de documents scientifiques de niveau recherche, publiés ou non, émanant des établissements d'enseignement et de recherche français ou étrangers, des laboratoires publics ou privés. 


\title{
Tensile mechanics of polymeric foam ribbons
}

\author{
A. Mouquet ${ }^{1,3}$, Y. Khidas ${ }^{2}$, X. Chateau ${ }^{1}$, T. Saison ${ }^{3}$, J.-Y. Faou ${ }^{3}$, and O. Pitois ${ }^{1, *}$ \\ ${ }^{1}$ Université Paris Est, Laboratoire Navier, UMR 8205 CNRS - École des Ponts ParisTech - IFSTTAR \\ cité Descartes, 2 allée Kepler, 77420 Champs-sur-Marne, France. \\ aymeric.mouquet@gmail.com; xavier.chateau@ifsttar.fr; olivier.pitois@ifsttar.fr \\ ${ }^{2}$ Université Paris Est, Laboratoire Navier, UMR 8205 CNRS - École des Ponts ParisTech - IFSTTAR \\ 5 bd Descartes, 77454 Marne-la-Vallée Cedex 2, France. \\ vacine.khidas@u-pem.fr \\ ${ }^{3}$ Saint-Gobain Research Paris, 39 Quai Lucien Lefranc, 93300 Aubervilliers, France \\ tamar.saison@saint-gobain.com; jean-yvon.faou@saint-gobain.com
}

\begin{abstract}
Whereas size reduction that accompanies new technologies calls for more complex and more constrained shapes for foams to be used as seals, ribbons or surface coatings, little is known about the mechanics of foam systems characterized by a potentially small number of pores across their thickness and possessing parietal or free surface pore layers. Elastic stiffness and rupture stress have been measured for open-cell foam ribbons produced thanks to a method allowing to tune finely and independently several crucial parameters: ribbon thickness, pore size and solid volume fraction. Results reveal that the longitudinal elastic stiffness of foam ribbons increases significantly as the number of pores across the ribbon thickness decreases within the range 1-10. This increase is understood through the contribution of the parietal layers with respect to the bulk layers. Similarly, rupture stress of foam ribbons characterized by narrow pore size distributions increases as a function of the ratio pore size/ribbon thickness. All others things being equal, the rupture stress is smaller polydisperse samples compared to monodisperse samples.
\end{abstract}

\section{Introduction}


Polymeric foams are widely used from years in many different fields thanks to their large specific surface area, low density, mechanical properties and thermal/acoustic insulation properties [1-4]. Size reduction that accompanies new technologies, e.g. microelectronics, calls for more complex and more constrained shapes for foams to be used as seals, ribbons or surface coatings. Actually, for damping purpose, thin foam layers need to be pasted on the surface of electronic elements, such as smartphone screens for example. Within those operating conditions, or during handing stages, such thin foam layers are likely to be subjected to strong compression, elongation or shear stresses, and they are expected to respond with appropriate damping and elastic properties at low or/and high strains. Although mechanics of bulk foam has been the subject of numerous studies, either through compression at both small and high strains [1,5-8] or through tension [9-12], mechanical properties of foam ribbons/coatings have not been studied so far.

Among fundamental questions raised by such a specific shaping of foams are those related to the effect of the potentially small number of bubbles (or pores) across the foam thickness. Note that here thin slices cut in bulk foam are not referred to, but instead, foams possessing parietal or free surface bubble layers are considered, i.e. foams that have been produced specifically with small thickness. With respect to bulk foam, such foams are expected to possess wall-induced structural order, whose range depends on bubble size distribution. For example, wall-bounded monodisperses bubbles are known to exhibit crystal-like structures over significant number of layers further away from the wall [13-15]. Such an order induces significant deviation of mechanical properties with respect to random assemblies of monodisperse bubbles [16,17]. A second potential issue comes from the intrinsic structure of the parietal bubble layers [18]. The corresponding mechanical feature, if it exists, could contribute significantly to the overall mechanical response of wall-bounded foam. Therefore, one question arises: what are the conditions, in terms of foam thickness and bubble size values, for the parietal contribution to be significant?

As far as we know, a study of such effects has never been performed before. Here, we focus on the mechanical behavior measured for open-cell polymeric foam ribbons within tensile conditions. In order to decouple completely the effects of both pore size and ribbon thickness, a dedicated foaming method is used to produce systems with fixed values for pore size, ribbon thickness, gas volume fraction and elastic stiffness of the solid matrix. Small strain oscillatory experiments are performed to measure the elastic stiffness of foam ribbons. Then high strain measurements are performed until rupture. As it 
will be shown in the following, the longitudinal tensile elasticity of foam ribbons exhibits a significant effect of the thickness-to-pore size ratio, regardless of the bubble size distribution, i.e. monodisperse or polydisperse. Such an effect is reasonably described by accounting for the contribution of the two parietal pore layers.

\section{Materials and methods}

\subsection{Raw materials}

A polyurethane dispersion (PUD) is used. It is made by mixing an aqueous suspension of $1 \mu \mathrm{m}$ surfactant-stabilized polyurethane particles with a cross-linker. The average particle volume fraction is $\varphi_{P U D}^{0} \approx 55 \%$ (45\% water). The density and the shear viscosity of the PUD are $1100 \mathrm{~kg} / \mathrm{m}^{3}$ and $\mu_{0} \approx$ 1 Pa.s (measured at low shear rate, i.e. $1 \mathrm{~s}^{-1}$ ) respectively. Surfactant used to stabilize the foam films is Tetradecyltrimethylammonium bromide (TTAB). It is used in aqueous solution at a concentration 10 g. $\mathrm{L}^{-1}$ (approximately tenfold the critical micelle concentration). The surface tension of the foaming solution is $\gamma \approx 0.04 \mathrm{~N} / \mathrm{m}$.

Making the foam ribbons involves coating first solid substrates with thin layer of PUD foam and then removing the foam layer. Peeling off from the substrate without damage was found to be allowed only silicone coated plastic substrates.

\subsection{Production of well-controlled polymer foam ribbons from PU dispersion}

The production method has been published elsewhere [19]. The main steps are presented in Fig. 1 and can be summarized as follows:

(1) Precursor aqueous foam is produced from the TTAB solution and nitrogen by using microfluidics technics. Such technics allow for monodisperse bubbles to be produced with targeted size $D_{b}$ ranging between $200 \mu \mathrm{m}$ and few millimeters. The typical production rate is $10 \mathrm{~mL} \cdot \mathrm{min}^{-1}$. Generated bubbles are continuously collected into a reservoir (vertical glass column) where the foam gas fraction was maintained to a constant value $\phi_{0}=0.92 \pm 0.02$. Note that foam coarsening turns out to be prevented over the duration of the production step, so monodisperse precursor aqueous foams were 
obtained. In addition, polydisperse foams were made out from $320 \mu \mathrm{m}$ monodisperse bubbles (obtained as described above), by letting time for the coarsening process to act in the reservoir.

(2) After production of the required precursor foam volume, the latter is mixed (in-line mixing) with the polyurethane dispersion to obtain fresh PUD foam. The gas volume fraction within the resulting PUD foam (and so the solid volume fraction $\phi_{S}$ in the final solid foam ribbon) can be adjusted by tuning the ratio of flow rates for the precursor foam $\left(Q_{p f}\right)$ and the polyurethane dispersion $\left(Q_{P U D}\right)$. It was shown in [19] that the final solid volume fraction $\phi_{S}$ can be related to the production parameters through: $\phi_{S} \cong 0.6 \times Q_{P U D} /\left(Q_{P U D}+Q_{p f}\right)$, within the range $0.08 \lesssim \phi_{S} \lesssim 0.3$.

(3) Then the PUD foam is solidified by evaporating the water from the fresh foam in a climatic chamber during $12 \mathrm{~h}$ at $\mathrm{T}=20^{\circ} \mathrm{C}$ and relative humidity $\mathrm{RH}=40 \%$. Note that the structure of the foam appeared to be already set after $30 \mathrm{~min}$ in the chamber, but complete water removal requires more time, i.e. $12 \mathrm{~h}$.

(4) The dried foam is then cured during 15 minutes at $150^{\circ} \mathrm{C}$ in order to activate the polymer crosslinking. Such curing conditions result from compromise made between cross-linking of the polymer matrix and temperature-induced partial collapse of the foam structure.

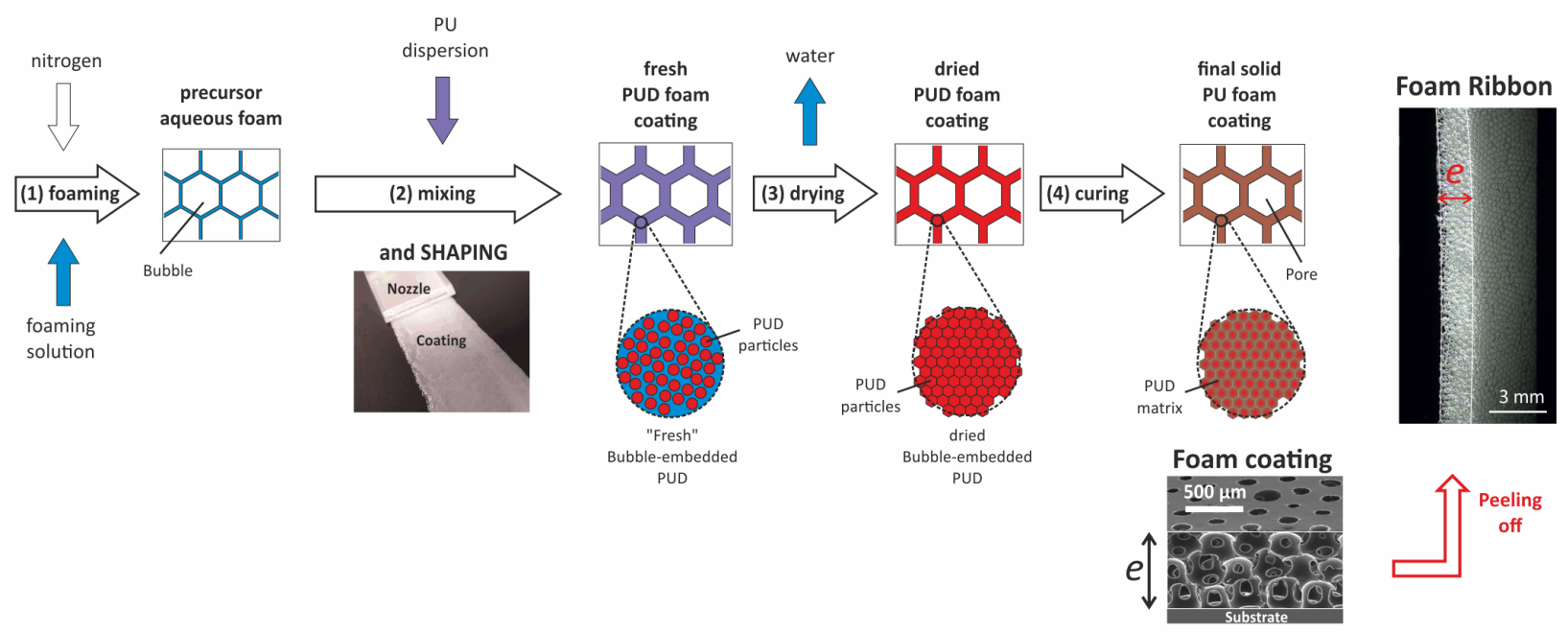

Figure 1: Sketch showing the main steps of the method used to produce solid polymer foams from aqueous polyurethane dispersion (PUD). (1) The precursor aqueous foam is made by using microfluidics. The bubble/pore size is set at this stage. (2) The precursor foam is mixed with the polyurethane dispersion. The PU content is set by volume flow rates of precursor foam and PUD. At this stage the shape of the ribbons is set by spreading the fresh PUD foam on a solid substrate. The thickness of the foam coating is set by the spreading velocity. (3) The fresh PUD foam is dried for $12 \mathrm{~h}$ in a climatic chamber. (4) The dried PUD foam is cured at $150^{\circ} \mathrm{C}$ for $15 \mathrm{~min}$ in order to activate the cross-linker. The resulting open-cell solid foam coating has pore size $D_{p}$ equal 
to bubble of the precursor foam, whereas thickness e and solid volume fraction $\phi_{s}$ have been set respectively at stages (2) and (3). Cutting and peeling off the foam coating allow foam ribbon samples (i.e. two free surfaces) to be obtained, such as the one presented to the right.

\subsection{Shaping of the PUD foam ribbons}

Foam shaping is made during step (2) described in the previous section (see also Fig. 1). A dedicated nozzle of width $30 \mathrm{~mm}$ is fed with the fresh PUD foam from the mixing device at a flow rate $Q_{p f}+Q_{P U D}$ and it is allowed to slide onto a silicone-coated plastic liner moved at constant velocity thanks to a motorized translation stage (the stage velocity $v$ can be set between $0.1 \mathrm{~mm} . \mathrm{s}^{-1}$ and to 40 $\mathrm{mm} . \mathrm{s}^{-1}$, with a precision of $\left.0.01 \mathrm{~mm} \cdot \mathrm{s}^{-1}\right)$. The thickness $e$ of the resulting foam layer was shown [19] to be tuned by adjusting the PUD foam flow rate $\left(Q_{p f}+Q_{P U D}\right)$ and/or the translation stage velocity $(v)$ :

$e \cong 50 \times\left(Q_{p f}+Q_{P U D}\right) / v$. Note that (i) although the gap of the nozzle was not found to set the foam thickness (as already said it is set mainly by $v$ for a given foam flow rate), foam layers with uniform thickness were obtained only for gaps ranging between $0.3 \mathrm{~mm}$ and $1.5 \mathrm{~mm}$; (ii) for monodisperse samples, layered structures were obtained (as explained in detail in the following), so the observed variation for the thickness corresponds to the stair-step curve of the proposed relation.

After a rest period of 24 hours, rectangular samples of surface area $S=6.1 \mathrm{~mm} \times 40 \mathrm{~mm}$ were cut in the foam coating by using a metallic punch form for die-cutting (Instron). Foam ribbon samples were obtained by removing (peeling off) those cuts from the liner. Ribbon thickness $e$ was measured by observation of their cross-section using optical microscopy (Zeiss SteREO Discovery V.8). Mass $m$ was measured for each sample using a precision scale, providing the sample's density: $\langle\rho\rangle=m /(S \times e)$. The solid volume fraction was deduced from the density of the PUD matrix $\rho_{S}:\left\langle\phi_{S}\right\rangle=\langle\rho\rangle / \rho_{S}$.

\subsection{Production PUD unfoamed ribbons}

Unfoamed PUD ribbons were produced from the PU dispersion by using a calibrated Mayer rod coater on liner. Resulting coating films were then dried at room temperature and cured in an oven at $150^{\circ} \mathrm{C}$ during $15 \mathrm{~min}$. Special attention was made for the resulting coating thickness to be of the same order of magnitude than the size of the foam network elements, i.e. between 50 and $100 \mu \mathrm{m}$. This is expected to ensure comparable curing conditions for both unfoamed coatings and foam coatings, and therefore comparable polymer cross-linking conditions. Moreover, as PU particles are densely packed 
together during water evaporation (i.e. after the coating shaping step), any residual anisotropy effect from the initial stretching of the PU dispersion is not expected. After a rest period of 24 hours, rectangular samples were cut similarly to what was done with the foam coatings, i.e. using a metallic punch form for die-cutting. Ribbons were obtained by peeling off the cuts from the liner. Ribbon's thickness was measured with a caliper before performing the mechanical test. With the same purpose, Scanning Electron Microscopy (Bruker quanta $400 \mathrm{FEI}$, tungsten filament, Pt coating $2 \mathrm{~nm}$ ) was used after the mechanical test. Density of the unfoamed ribbons was measured to be $\rho_{S}=1.1 \mathrm{~g} / \mathrm{cm}^{3}$.

\subsection{Pore morphology and foam structure}

Several samples of size close to $1 \mathrm{~cm} \times 1 \mathrm{~cm}$ were cut within the ribbons and were studied using Scanning Electron Microscopy (Bruker quanta $400 \mathrm{FEI}$, tungsten filament, Pt coating 2nm) and X-ray tomography (EasyTom RX Solutions). Thanks to the difference in X-ray absorption between PU and air, it was possible to reconstruct contrasted 3D images, i.e. stacks of slices parallel to the ribbon's plane, where the pores were reasonably separated from the polymer matrix. The voxel size was close to $5 \mu \mathrm{m}$. For each slice of height $z$ and thickness equal to one voxel, the solid volume fraction $\phi(z)$ was determined by dividing the number of voxels corresponding to the polymer matrix are by the total number of voxel in the slice. The pores were identified by using the 3D Distance Transform Watershed from the MorphoLibJ plugin [20] in the open source software Fiji [21] (see [19] for more details) in order to measure the volume $V_{p}$ for each pore. The pore size distribution of our samples was therefore characterized from the equivalent diameter under the assumption of spherical pore shape $\left(D_{p}=\right.$ $\left.\sqrt[3]{6 V_{p} / \pi}\right)$ 

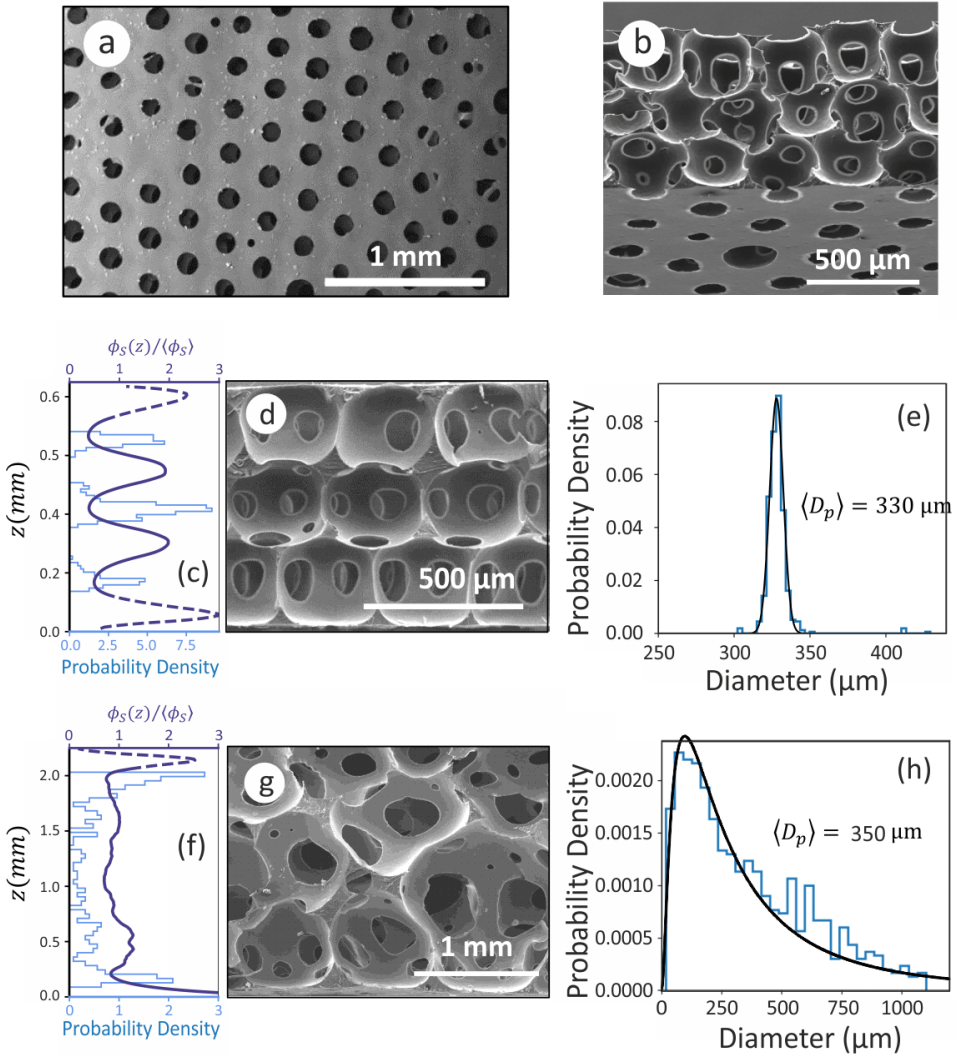

Figure 2: Typical examples of foam ribbon samples made in this study. (a) SEM picture showing the top free surface, where the pores of the top layer are open (monodisperse sample, pore diameter: $330 \mu \mathrm{m})$. (b) SEM picture showing the pore layer that was initially in contact with the substrate as well as the bulk pores (monodisperse sample, pore diameter: 330 $\mu m$ ). (c-e) monodisperse sample $\left(e=830 \mu \mathrm{m},\left\langle D_{p}\right\rangle=330 \mu \mathrm{m},\left\langle\phi_{s}\right\rangle=0.14\right)$ : (c) Typical solid volume fraction profile $\phi_{s}(z)$ and pore position probability density measured across the ribbon's thickness $e$. Dashed curves indicate areas for which solid fraction should be considered as an "apparent" value due to the "roughness" at the free top surface of the samples. (d) SEM picture showing a side view of the sample. (e) number weighted pore diameter distribution. The solid line represents a normal distribution with parameters $m_{n}=330$ and $\sigma_{n}=4.8$ (respectively the mean and the standard deviation of the size distribution). (f-h) polydisperse sample ( $\left.e=2250 \mu \mathrm{m},\left\langle D_{p}\right\rangle=350 \mu \mathrm{m},\left\langle\phi_{s}\right\rangle=0.13\right)$ : (f) Typical solid volume fraction profile $\phi_{s}(z)$ and pore position probability density measured across the ribbon's thickness e. (g) SEM picture showing a side view of the sample. (h) number weighted pore diameter distribution. The solid line represents a log-normal distribution with parameters $m_{l n}=5.65$ and $\sigma_{l n}=1$ (respectively the mean and the standard deviation of the size's natural logarithm).

Typical examples for the structure of the foam ribbons are presented in Fig. 2. The so-called "monodisperse" samples are characterized by a narrow pore size distribution (Fig. 2e). They show triangular arrangement of pores at both their top and bottom surfaces (see Fig.2a,b) as well as layered arrangement of pores across their thickness (see Fig. 2b,d). This is confirmed by both density profiles and bubble positions (such as those presented in Fig. 2c) measured across ribbons' thickness. This structure results from the combination of two effects: planar wall boundary effect and packing properties of 
monodisperse assemblies of spheres. Note that such a structure was observed for samples counting up to six-seven pore sizes across their thickness, but thicker samples showed progressive loss of the layered structure, except for areas close to the two planar boundaries. As expected, polydisperse samples do not exhibit such a structure, which is shown in Figs $2 \mathrm{f}-\mathrm{h}$. Note that all the studied samples are open-cell foams (see [article 1] for more details about the size of the apertures connecting the pores).

\subsection{Mechanics}

Dynamical Mechanical Analyzer (DMA Q800 - TAinstrument) was used to measure the tensile elastic stiffness of both foam ribbons and solid PUD through oscillatory tension tests. Samples were placed between two tension jaws as a static force was applied in order to maintain a tension state corresponding to initial strain approximately equal to $0.2 \%$. Sinusoidal deformations were then superimposed to the static deformation in order to measure the tensile elastic stiffness within different conditions: (1) different strain amplitudes were applied at fixed frequency $(1 \mathrm{~Hz})$ and, (2) different frequencies were studied at constant strain amplitude (0.01\%). High strain measurements were performed by using a tensile testing machine (Instron 3365) with a $50 \mathrm{~N}$ force sensor and sample placed between two tension jaws. Samples were stretched at a constant strain rate equal to $1.25 \times 10^{-3} \mathrm{~s}^{-1}$ until rupture occurred.

\section{Small strain oscillatory tension}

Typical results for the storage $\left(E^{\prime}\right)$ and loss $\left(E^{\prime \prime}\right)$ moduli of PU samples are presented on Fig. 3. First of all, the mechanical behavior of both unfoamed ribbons and foam ribbons can be reasonably described as linear viscoelasticity due to the linear regime observed for both $E^{\prime}$ and $E^{\prime \prime}$ at small strain (see Figs $3 a, c)$. We recall that within our solicitation conditions, a constant tension stress is applied as corresponding to a deformation equal to $0.2 \%$, so deformations larger than this value cannot be studied (shaded range in Figs $3 a, c$ ). In the following, reported values for $E^{\prime}$ will correspond to a strain amplitude $\varepsilon=0.01 \%$. It can be seen that (i) $E^{\prime}>E^{\prime \prime}$ for both unfoamed ribbon and foam ribbon, (ii) solicitation frequency has significant effect on those values: $E^{\prime}$ is increased by $50 \%$ as frequency is increased by two decades (see Figs $3 b, d$ ), and (iii) values measured for foam ribbons $E_{r}^{\prime}$ are almost one order of magnitude smaller than values measured for unfoamed ribbons $E_{S}^{\prime}$, because of the small amount of PU matrix in the foam. 

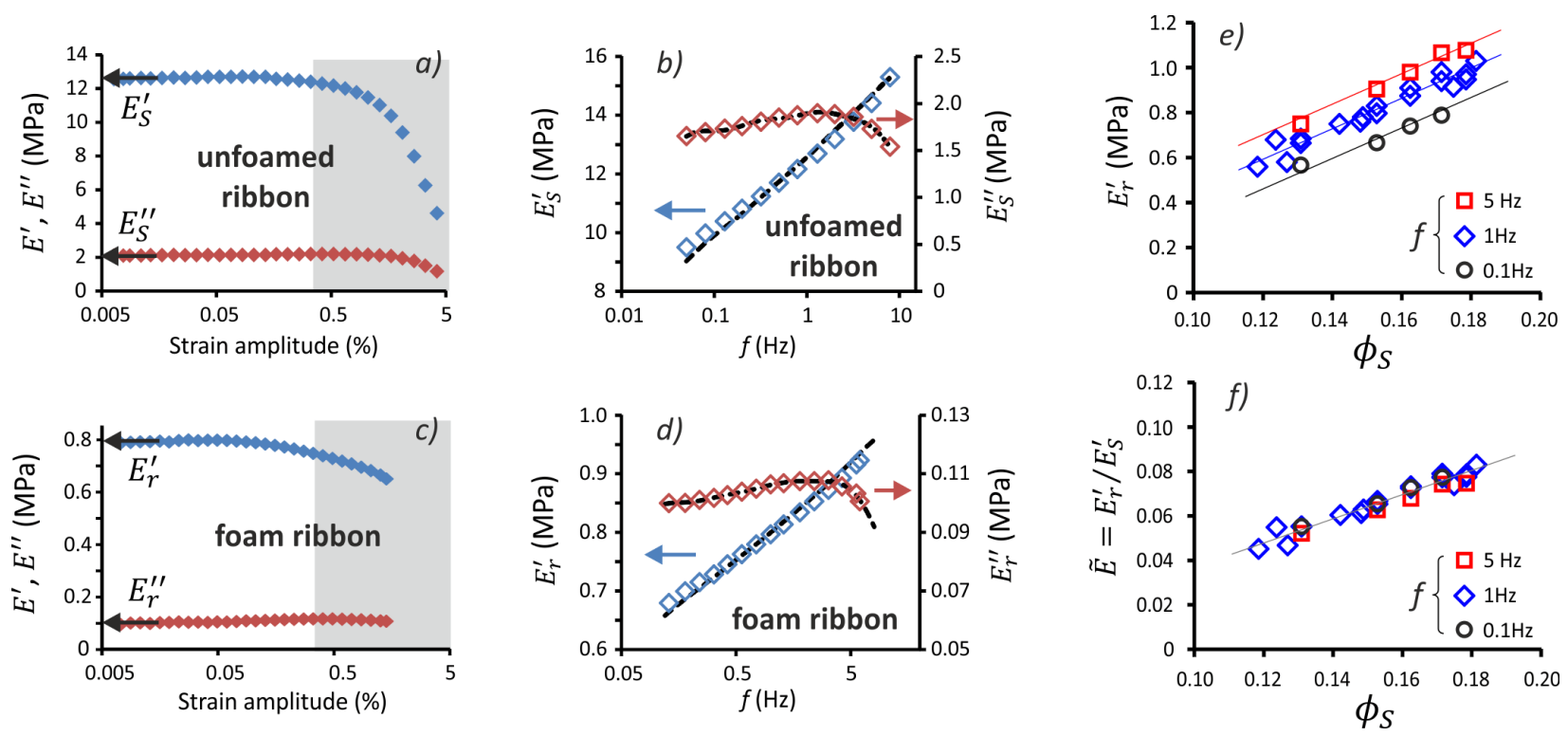

Figure 3: Typical measured storage $\left(E^{\prime}\right)$ and loss $\left(E^{\prime \prime}\right)$ moduli for both unfoamed ribbons and foam ribbons. (a) Unfoamed ribbons: effect of strain amplitude for a fixed frequency $(1 \mathrm{~Hz})$. The elastic stiffness measured at small strain amplitude is represented by $E_{S}^{\prime}$. (b) Unfoamed ribbons: effect of frequency for fixed strain amplitude $(0.01 \%)$. The dotted lines correspond to the generalized Maxwell model (see main text for details) with parameters: $E_{0}=7.64 \mathrm{MPa}, E_{1}=2.33 \mathrm{MPa}, E_{2}=1.93 \mathrm{MPa}, E_{3}=$ $1.81 \mathrm{MPa}, E_{4}=2.52 \mathrm{MPa}, \tau_{1}=0.02 \mathrm{~s}, \tau_{2}=0.12 \mathrm{~s}, \tau_{3}=0.55 \mathrm{~s}, \tau_{4}=3.37 \mathrm{~s}$. (c) $P U$ foam ribbon $\left(\phi_{s}=0.15, e=0.8 \mathrm{~mm}, D_{p}=\right.$ $340 \mu \mathrm{m})$ : effect of strain amplitude for a fixed frequency $(1 \mathrm{~Hz})$. The elastic stiffness measured at small strain amplitude is represented by $E_{r}^{\prime}$. (d) PU foam ribbon $\left(\phi_{S}=0.15, e=0.8 \mathrm{~mm}, D_{p}=340 \mu \mathrm{m}\right)$ : effect of frequency for fixed strain amplitude $(0.01 \%)$. The dotted lines correspond to the generalized Maxwell model (see main text for details) with parameters: $E_{0}=$ $0.62 \mathrm{MPa}, E_{1}=0.25 \mathrm{MPa}, E_{2}=0.09 \mathrm{MPa}, E_{3}=0.08 \mathrm{MPa}, E_{4}=0.09 \mathrm{MPa}, \tau_{1}=0.013 \mathrm{~s}, \tau_{2}=0.08 \mathrm{~s}, \tau_{3}=0.29 \mathrm{~s}, \tau_{4}=1.34 \mathrm{~s}$. (e) Elastic stiffness measured at small strain amplitude for foam ribbons ( $e=0.8 \mathrm{~mm}, D_{p}=340 \mu \mathrm{m}$ ) as a function of solid volume fraction $\phi_{S}$ and for several solicitation frequencies $f$ within the range 0.1-5 Hz. Solid lines are guides for the eye. (f) Dimensionless elastic stiffness for foam ribbons, i.e. data from Fig. 3 e for $E_{r}^{\prime}\left(\phi_{S}, f\right)$ divided by $E_{S}^{\prime}(f)$, as a function of solid volume fraction $\phi_{S}$ and for several solicitation frequencies $f$ within the range 0.1-5 Hz. Solid line is a guide for the eye.

Viscoelasticity is modeled by using the generalized Maxwell model with $n$ Maxwell elements in parallel with a spring [22]. Each Maxwell element $i$ has characteristic time $\tau_{i}=\eta_{i} / E_{i}$, where $E_{i}$ and $\eta_{i}$ are respectively the elastic modulus and the viscosity. For oscillatory tension, storage and loss moduli are respectively given by relations: $E^{\prime}(\omega)=E_{0}+\sum_{i=1}^{n} E_{i} \frac{\omega^{2} \tau_{i}{ }^{2}}{1+\omega^{2} \tau_{i}{ }^{2}} \quad$ and $E^{\prime \prime}(\omega)=\sum_{i=1}^{n} E_{i} \frac{\omega \tau_{i}}{1+\omega^{2} \tau_{i}{ }^{2}}$ with $\omega=2 \pi f$ the pulsation. Figs $3 b, d$ show that good agreement can be found within the investigated frequency range by using four characteristic times $\tau_{i}$. The latter were found to be very close for both unfoamed ribbons and foam ribbons, whatever the thickness and the gas volume fraction of the foam ribbons, which means that viscoelasticity observed for foam ribbons can be understood mainly by the viscoelastic properties of the PU matrix. Therefore, it turns out to be appropriate to make dimensionless the measured elastic modulus for foam ribbons $E_{r}^{\prime}(f)$ at frequency $f$ by dividing by the value measured 
for the unfoamed ribbons $E_{S}^{\prime}(f)$, i.e. $\tilde{E}=E_{r}^{\prime}(f) / E_{S}^{\prime}(f)$. Such a procedure is presented in Fig. 3 for foam ribbons characterized by different solid volume fractions $\phi_{S}$ : the effect of the solicitation frequency observed in Fig. 3e for $E_{r}^{\prime}(f)$ is removed when considering instead $\tilde{E}$ (see Fig. 3f). In the following, effects of parameters $\phi_{S}, D_{p}$ and $e$ will be analyzed through the variation of $\tilde{E}=E_{r}^{\prime}(1 \mathrm{~Hz}) / E_{S}^{\prime}(1 \mathrm{~Hz})$.

Fig. 4 shows measured $\tilde{E}$ values for both monodisperse and polydisperse foam ribbons as a function of solid volume fraction $\phi_{S}$. Effect of ribbon thickness is presented in Fig. 4a for a fixed pore size value. Whatever the solid volume fraction, $\tilde{E}$ decreases as a function of ribbon thickness. As expected, $\tilde{E}$ increases as a function of $\phi_{S}$, whatever the ribbon thickness. Such strengthening of foam with the amount of solid is usually describes by power laws [1], i.e. $\tilde{E}=a \phi_{S}^{b}$. Such power law curve has been fitted to our data, providing almost constant values for the exponent: $b \cong 1.3-1.5$. Those values differ significantly from the classical Gibson \& Ashby behavior for open-cell foam, which is described by $b=2$. The effect of ribbon thickness-to-pore size ratio is presented in Fig. 4b. It is revealed that pore size also has significant effect on $\tilde{E}$. This is a crucial difference with respect to bulk foam elasticity [1]. Results for the effect of thickness of polydisperse foam ribbons are presented in Fig. 4c. Similarly to previous observations with monodisperse samples, $\tilde{E}$ is found to increase as a function of $\phi_{S}$ but to decrease as a function of ribbon thickness. 

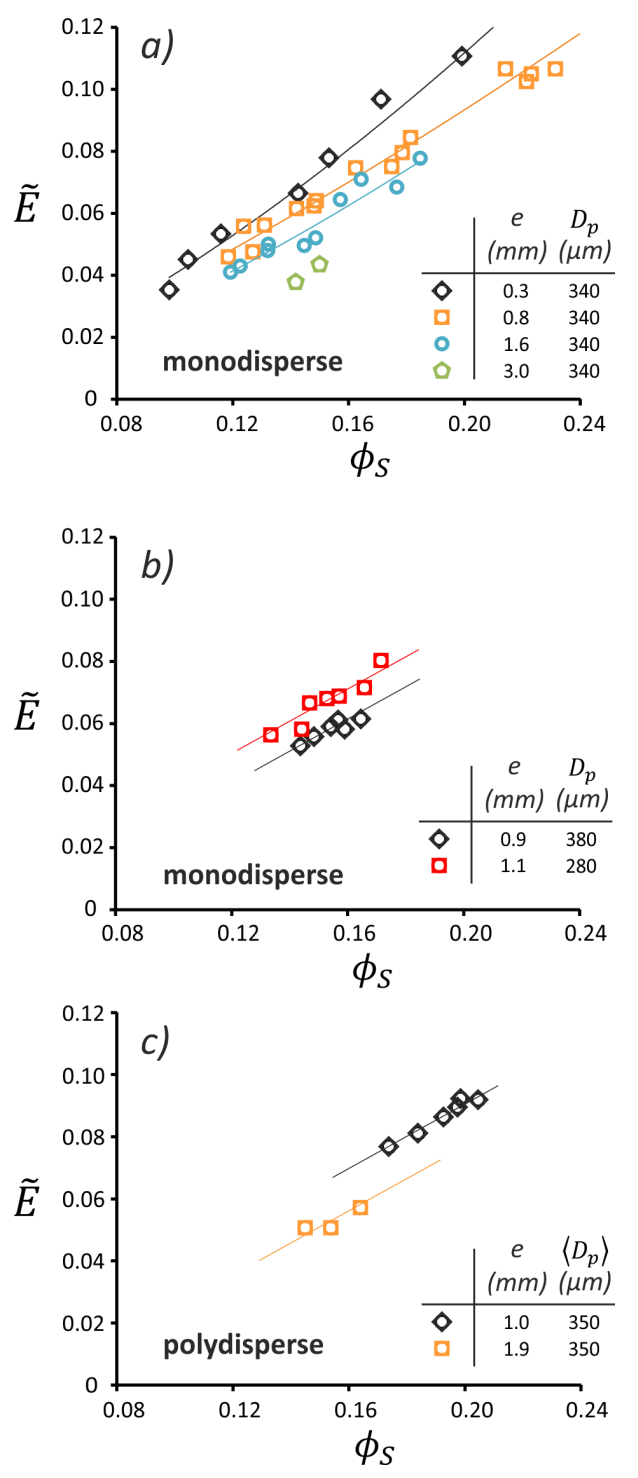

Figure 4: Dimensionless tensile stiffness measured for foam ribbons as a function of solid volume fraction, for different pore sizes $D_{p}$ and ribbon thickness $e$ (as indicated in the figures). (a) Monodisperse foam: Effect of ribbon thickness for a fixed pore size. Solid lines correspond to fitted power laws, i.e. $\tilde{E}=a \phi_{S}^{b}$, with $a=1.08$ and $b=1.46$ for $e=0.3 \mathrm{~mm}, a=0.74$ and $b=1.28$ for $e=$ $0.8 \mathrm{~mm}, a=0.86$ and $b=1.48$ for $e=1.6 \mathrm{~mm}$. (b) Monodisperse foam: Effect of pore size for a fixed ribbon thickness. Solid lines are guides for the eye. (c) Polydisperse foam: Effect of ribbon thickness.

\section{High strain mechanics}

Fig. 5a presents typical stress-strain curves obtained for stretching of foam ribbons up to rupture at stress $\sigma_{r}^{*}$. Note that rupture occurs for very large deformations, i.e. typically 500\%. Moreover, the rupture stress increases while the rupture strain decreases as a function of solid volume fraction. Such a behavior is classically observed for bulk elastomeric foams [1]. Fig. 5b shows measured $\sigma_{r}^{*}$ values for 
both monodisperse and polydisperse foams with same pore size (i.e. $D_{p}=340 \mu \mathrm{m}$ and $\left\langle D_{p}\right\rangle=350 \mu \mathrm{m}$ respectively for monodisperse and polydisperse samples) but different ribbon thicknesses: thinner ribbons have higher $\sigma_{r}^{*}$ values. This is similar to the above-reported behavior for the elastic stiffness. We stress however that the thinnest polydisperse ribbons represent exception to that behavior due to anomalous stress-strain curves (not shown) and early rupture.
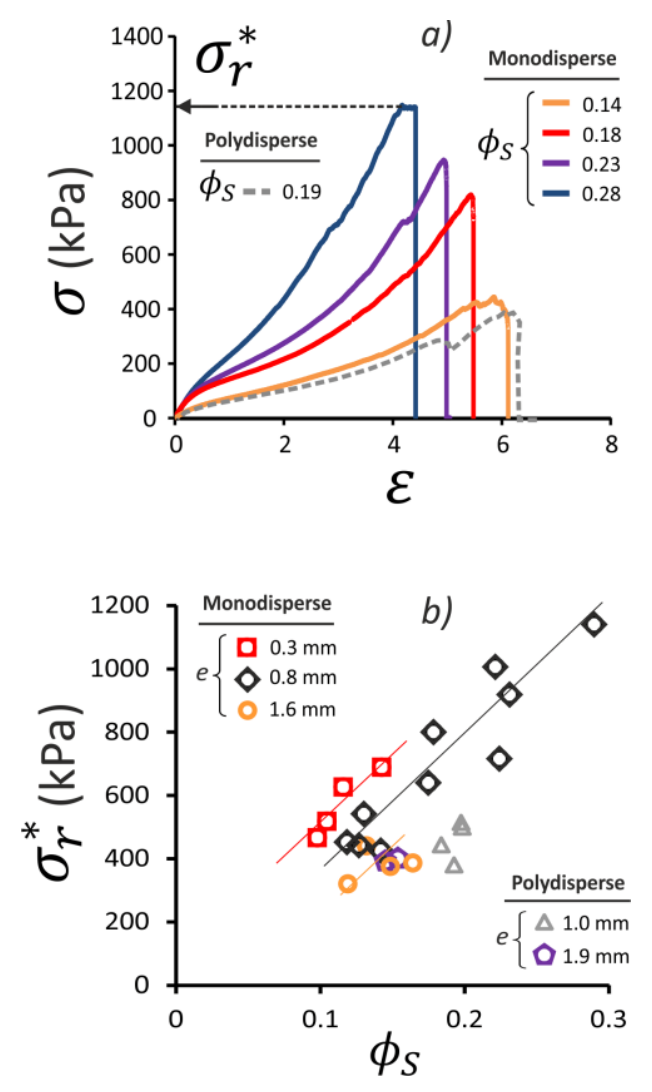

Figure 5: Large strain deformations of foam ribbons. (a) Typical stress-strain curves obtained for foam ribbons with thickness $e=$ $810 \pm 40 \mu \mathrm{m}$. The pore size is $D_{p}=340 \mu \mathrm{m}$ for monodisperse samples and $\left\langle D_{p}\right\rangle=350 \mu \mathrm{m}$ for polydisperse samples. Note that rupture is observed for stress $\sigma_{r}^{*}$. (b) Rupture stress $\sigma_{r}^{*}$ as a function of $\phi_{S}$ for both monodisperse $\left(D_{p}=340 \mu \mathrm{m}\right)$ and polydisperse $\left(\left\langle D_{p}\right\rangle=350 \mu \mathrm{m}\right)$ foam ribbons with different thicknesses (as indicated). Solid strait lines are guides for the eye.

\section{Discussion}

Results presented in the previous section have shown that, in addition to the effect related to the amount of solid matrix, mechanics of foam ribbons depends on both pore size and ribbon thickness. This 
is the major difference with respect to classical bulk foam, so the following discussion aims to understand this behavior.

Density profiles have shown that significant amount of solid matrix is contained within the two parietal layers. This property is related to the specific structure of parietal layers, i.e. size and orientations of the parietal struts. Therefore, a specific elastic modulus $E_{w}$ should be attributed to each parietal layer over a thickness $e_{w}$. As a first attempt to model the reported behavior, a simple combination of 3 Hookean springs in parallel is assumed: 2 springs accounting for the two parietal layers and 1 spring for the core (bulk) material. Then the overall elastic modulus of the ribbons reads $E_{r}=\left[2 E_{w} e_{w}+E_{\text {bulk }}\left(e-2 e_{w}\right)\right] / e$, where $E_{\text {bulk }}$ is the elastic modulus of the bulk foam. By introducing the proportion of parietal layers $x_{w}=2 e_{w} / e$ and by dividing by $E_{b u l k}$, the elastic modulus becomes:

$$
\left.\frac{E_{r}}{E_{\text {bulk }}} \approx 1+x_{w}\left(\frac{E_{w}}{E_{\text {bulk }}}-1\right) \quad \text { (eq. } 1\right)
$$

In order to compare equation 1 with experimental data, one has to set values for $e_{w}$ (or equivalently $\left.x_{w}\right), E_{w}$ and $E_{b u l k}$. Thickness $e_{w}$ is expected to be set by the pore size, so in the following $x_{w}=\left\langle D_{p}\right\rangle / e$ is used. As already mentioned, the elastic modulus of bulk foam depends on solid volume fraction only, as described by power laws, e.g. $\tilde{E}_{b u l k}=a \phi_{s}^{b}$. Our data are reasonably described by power laws with exponent $b \cong 1.3-1.5$. This exponent is smaller than the value corresponding to the classical behavior reported by Gibson \& Ashby [1], i.e. $b \cong 2$. Such behavior is obtained for open-cell foam at low solid content, where the structure is made of solid struts of length $\ell \sim D_{p}$ and width $t \sim D_{p} \phi_{s}^{1 / 2}$. By assuming that foam deformation is associated to bending of those struts, one obtains that the reduced elastic modulus is $\tilde{E}_{\text {bulk }} \sim t^{4} / D_{p}^{4} \sim \phi_{s}^{2}$. However, assumptions made to derive such a relation are not justified at higher solid content, i.e. $\phi_{s}>0.1$. In particular, significant amount of solid is contained within the nodes connecting the struts, which is expected to lead to a different behavior. For example, the elastic modulus of realistic numerical foams has been shown to be described by $\widetilde{E}_{b u l k}=0.7 \Phi_{S}^{1.45}$ for $\phi_{S}$ values within the range 0.05-0.2 [23]. Actually, the precise choice made for $\tilde{E}_{\text {bulk }}$ is not crucial to highlight the contribution of parietal layers in the foam ribbons. In the following, we assume that $\tilde{E}_{\text {bulk }}=a \Phi_{s}^{1.45}$ with $a$ close to 0.7 . For the sake of simplicity, we assume that parietal layers undergo the same type of geometrical evolution in terms of thickness and length of the struts as a function of $\phi_{s}$, i.e. $\tilde{E}_{w}\left(\phi_{s}\right)=$ $w \phi_{s}^{1.45}$, where $w$ is a coefficient to be obtained by comparison of equation 1 with our data. Such a 
comparison is presented in Fig. 6, where $E_{r} / E_{\text {bulk }}$ is plotted against $1 / x_{w}$ (we stress that $E_{r} / E_{b u l k} \equiv$ $\tilde{E} / \tilde{E}_{\text {bulk }}$ where experimental values for $\tilde{E}$ are shown in Fig. 4 ). Parameters $a=0.62$ and $w=1.3$ (i.e. $w / a \cong 2$ ) have been fitted in order to make all the data (both monodisperse and polydisperse samples) collapse on the theoretical curve. This means that the reduced excess modulus $\left(E_{r}-E_{\text {bulk }}\right) / E_{\text {bulk }}$ provided by parietal layers is approximately equal to $\left\langle D_{p}\right\rangle / e$. As a consequence, the elastic modulus of foam ribbons decreases from $E_{r} \cong 2 E_{\text {bulk }}$ for $\left\langle D_{p}\right\rangle / e \cong 1$, down to $E_{r} \cong E_{\text {bulk }}$ for $\left\langle D_{p}\right\rangle / e \rightarrow 0$.

Rupture of foams ribbons is expected to be described with the same approach, i.e. $\sigma_{r}^{*} / \sigma_{b u l k}^{*}=$ $1+x_{w}(w / a-1)$. The inset in Fig. 6 shows that indeed good agreement can be found for monodisperse samples using $w / a=2.1, x_{w}=\left\langle D_{p}\right\rangle / e$ and $\sigma_{b u l k}^{*} \cong 3500 \phi_{s}^{1.2}$. As already said, very thin polydisperse samples exhibit anomalous rupture behavior. Actually, Fig. 6 shows that polydisperse samples characterized by small $\left\langle D_{p}\right\rangle / e$ values have rupture stress $\sigma_{r}^{*}$ smaller than $\sigma_{b u l k}^{*}$. This could be understood by the fact that within polydisperse systems, pores with size significantly larger than the mean pore size act as defects in thin ribbons, i.e. hole bridging the two parietal layers, and initiate early rupture. Consequently, narrow pore size distributions appear to be preferable for ribbons characterized by small $\left\langle D_{p}\right\rangle / e$ values.

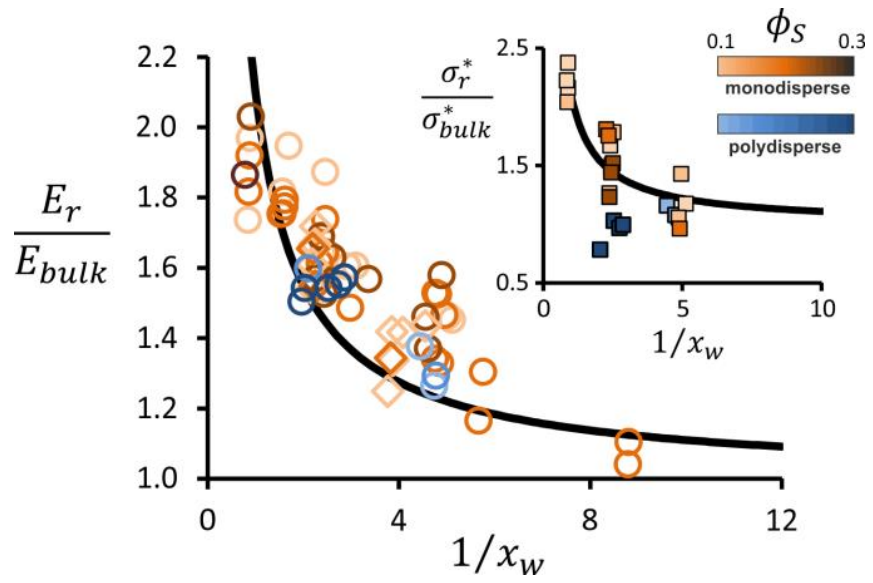

Figure 6: Evolution of the elastic stiffness of foam ribbons $\boldsymbol{E}_{\boldsymbol{r}}$ as a function of the thickness-to-pore size ratio $\mathbf{1} / \boldsymbol{x}_{\boldsymbol{w}}=\boldsymbol{e} /\left\langle\boldsymbol{D}_{\boldsymbol{p}}\right\rangle$. Presented data correspond to those of Fig. 4 (circles Fig. 4a, diamonds Fig. 4 b). Solid fractions $\boldsymbol{\phi}_{s}$ is indicated by the color scales. The solid line corresponds to the following relation: $\boldsymbol{E}_{r} / \boldsymbol{E}_{\text {bulk }}=\mathbf{1}+\mathbf{1 . 1}\left\langle\boldsymbol{D}_{\boldsymbol{p}}\right\rangle / \boldsymbol{e}$ with $\boldsymbol{E}_{\text {bulk }}=\mathbf{0 . 6 2} \phi_{s}^{\mathbf{1 . 4 5}}$. Inset: Evolution of the rupture stress $\boldsymbol{\sigma}_{\boldsymbol{r}}^{*}$ of foam ribbons as a function of the thickness-to-pore size ratio $1 / \boldsymbol{x}_{\boldsymbol{w}}=\boldsymbol{e} /\left\langle\boldsymbol{D}_{\boldsymbol{p}}\right\rangle$. Solid fractions $\boldsymbol{\phi}_{\boldsymbol{s}}$ is indicated by the color scales. The solid line corresponds to the following relation: $\sigma_{r}^{*} / \sigma_{\text {bulk }}^{*}=\mathbf{1}+\mathbf{1} . \mathbf{1}\left\langle\boldsymbol{D}_{\boldsymbol{p}}\right\rangle / \boldsymbol{e}$ with $\boldsymbol{\sigma}_{\text {bulk }}^{*}=$ $3500 \phi_{s}^{1.2}$. 


\section{Conclusion}

Elastic stiffness and rupture stress have been measured for foam ribbons. Our production method allowed to tune finely several crucial parameters: thickness, pore size and solid volume fraction. Results have shown that, in contrast to mechanics of bulk foam, mechanics of foam ribbons depends on pore size for fixed ribbon thickness. It was shown that the elastic stiffness is governed by the ratio of ribbon thickness-to-pore size. More precisely, the elastic stiffness increases as the size ratio decreases, and the relative strengthening reaches $100 \%$ when the size ratio is close to unity. This effect has been attributed the parietal layers, whose relative contribution increases as the size ratio decreases. Such a behavior has been observed for both monodisperse and polydisperse samples. For rupture stress, monodisperse samples follow the same behavior than for the elastic stiffness. On the other hand, polydisperse samples have shown anomalous early rupture behavior at small size ratio. It is suggested that pores with size significantly larger than the mean pore size act as defects in those thin ribbons. Consequently, narrow pore size distributions appear to be preferable for ribbons characterized by small size ratio.

\section{Data availability}

The raw/processed data required to reproduce these findings cannot be shared at this time due to technical or time limitations.

\section{References}

[1] L.J. Gibson, M.F. Ashby, Cellular Solids: Structure and Properties, Cambridge University Press, Cambridge, 1997.

[2] R. Verdejo, R. Stämpfli, M. Alvarez-lainez, S. Mourad, M.A. Rodriguez-perez, P.A. Brühwiler, Enhanced acoustic damping in flexible polyurethane foams filled with carbon nanotubes, Composites Science and Technology. 69 (2009) 1564-1569. doi:10.1016/j.compscitech.2008.07.003.

[3] S. Liu, J. Duvigneau, G.J. Vancso, Nanocellular polymer foams as promising high performance thermal insulation materials, European Polymer Journal. 65 (2015) 33-45. doi:10.1016/j.eurpolymj.2015.01.039.

[4] R.M. Christensen, Mechanics of cellular and other low density materials, Int. J. Solids Struct. 37 (2000) 93-104.

[5] W.E. Warren, A.M. Kraynik, The Linear Elastic Properties of Open-Cell Foams, Journal of Applied 
Mechanics. 55 (1988) 341-346.

[6] H.X. Zhu, N.J. Mills, Modelling the creep of open-cell polymer foams, Journal of the Mechanics and Physics of Solids. 47 (1999) 1437-1457. doi:10.1016/S0022-5096(98)00116-1.

[7] N.J. Mills, H. Zhu, The high strain compression of closed-cell polymer foams, Journal of the Mechanics and Physics of Solids. 47 (1999) 669-695. doi:10.1016/S0022-5096(98)00007-6.

[8] P. Viot, F. Beani, J.L. Lataillade, Polymeric foam behavior under dynamic compressive loading, Journal of Materials Science. 40 (2005) 5829-5837. doi:10.1007/s10853-005-4998-5.

[9] G. Pampolini, G. Del Piero, Strain localization in open-cell polyurethane foams: Experiments and theoretical model, Journal of Mechanics of Materials and Structures. 3 (2008) 969-981.

doi:10.2140/jomms.2008.3.969.

[10] M.E. Kabir, M.C. Saha, S. Jeelani, Tensile and fracture behavior of polymer foams, Materials Science and Engineering A. 429 (2006) 225-235. doi:10.1016/j.msea.2006.05.133.

[11] J. Lederman, The prediction of the tensile properties of flexible foams, Journal of Applied Polymer Science. 15 (1971) 693-703. doi:10.1002/app.1971.070150314.

[12] V. Shulmeister, M.W.D. Van der Burg, E. Van der Giessen, R. Marissen, A numerical study of large deformations of low-density elastomeric open-cell foams, Mechanics of Materials. 30 (1998) 125140. doi:10.1016/S0167-6636(98)00033-7.

[13] L. Bragg, J.F. Nye, A Dynamical Model of a Crystal Structure, Proceedings of the Royal Society of London. Series A. 190 (1947) 474-481.

http://web.mit.edu/mikejd/previously_online/aices_mit_2009/Bragg-bubble_raft.pdf (accessed June 18, 2019).

[14] A. Van Der Net, W. Drenckhan, D. Weaire, S. Hutzler, The crystal structure of bubbles in the wet foam limit, Soft Matter. 2 (2006) 129-134. doi:10.1039/b515537a.

[15] R. Höhler, Y.Y.C. Sang, E. Lorenceau, S. Cohen-Addad, Osmotic pressure and structures of monodisperse ordered foam, Langmuir. 24 (2008) 418-425. doi:10.1021/la702309h.

[16] L. Gong, S. Kyriakides, W.Y. Jang, Compressive response of open-cell foams. Part I: Morphology and elastic properties, International Journal of Solids and Structures. 42 (2005) 1355-1379. doi:10.1016/j.ijsolstr.2004.07.023.

[17] W.Y. Jang, A.M. Kraynik, S. Kyriakides, On the microstructure of open-cell foams and its effect on elastic properties, International Journal of Solids and Structures. 45 (2008) 1845-1875. doi:10.1016/j.ijsolstr.2007.10.008.

[18] E. Forel, E. Rio, M. Schneider, S. Beguin, D. Weaire, S. Hutzler, W. Drenckhan, The surface tells it all: relationship between volume and surface fraction of liquid dispersions, Soft Matter. 12 (2016) 8025-8029. doi:10.1039/C6SM01451H.

[19] A. Mouquet, Y. Khidas, T. Saison, J.-Y. Faou, O. Pitois, Well-controlled foam-based solid coatings, Soft Matter. 15 (2019) 5084-5093. doi:10.1039/C9SM00786E.

[20] D. Legland, I. Arganda-Carreras, P. Andrey, MorphoLibJ: integrated library and plugins for 
mathematical morphology with ImageJ, Bioinformatics. 32 (2016) 3532-3534.

doi:10.1093/bioinformatics/btw413.

[21] J. Schindelin, I. Arganda-Carreras, E. Frise, V. Kaynig, M. Longair, T. Pietzsch, S. Preibisch, C.

Rueden, S. Saalfeld, B. Schmid, J.-Y. Tinevez, D.J. White, V. Hartenstein, K. Eliceiri, P. Tomancak, A. Cardona, Fiji: an open-source platform for biological-image analysis, Nature Methods. 9 (2012) 676-682. doi:10.1038/nmeth.2019.

[22] I.M. Ward, J. Sweeney, Mechanical properties of solid polymers., Third edit, 2013. https://books.google.fr/books?hl=fr\&lr=\&id=sQNHQMYC1IC\&oi=fnd\&pg=PR5\&dq=Mechanical+Properties+of+Solid+Polymers,+l.+Ward+\%26+ J.+Sweeney\&ots=iFe3bU9izm\&sig=-YbD0ez2GJ5P5iE02BBmKokDpAo\#v=onepage\&q=Mechanical Properties of Solid Polymers\%2C I. W (accessed June 18, 2019).

[23] C. Lusso, X. Chateau, Numerical Modeling of Disordered Foam in 3D: Effective Properties by Homogenization., in: M. Vandamme, P. Dangla, J.-M. Pereira, S. Ghabezloo Editors, Poromechanics VI, 2017: pp. 1347-1354. 